\title{
MIGRATION SANS LA FAMILLE ET CONSTRUCTION DE L'ESPACE FAMILIAL EN SITUATION DE CONTRAINTE. LE CAS DES PERSONNES AGEES NEES EN AFRIQUE DU NORD-OUEST ET RESIDANT EN FRANCE
}

\section{YACINE ALAHYANE}

\author{
UNIVERSITÉ PAUL-VALÉRY MONTPELlier 3
}

RESUMEN: Este artículo tiene como objetivo analizar la construcción del espacio familiar en los ancianos nacidos en el norte y el oeste de África y que residen en Francia, lejos de su familia nuclear que vive en el país de origen. Busca comprender cómo esta construcción se lleva a cabo a lo largo de la trayectoria individual y familiar y resulta tanto de restricciones como de estrategias espaciales. Desarrolla la historia de esta migración a través del estado construido de "single geográfico" y muestra cómo esto impacta a la familia en su configuración espacial, organización y relaciones con el espacio.

PALABRAS CLAVE: Geografía, familia, migración, trayectoria, celibato

\section{NON-FAMILY MIGRATION AND THE CONSTRUCTION OF A FAMILY SPACE IN A SITUATION OF CONSTRAINT. THE CASE OF OLD PEOPLE BORN IN NORTHWEST AFRICA AND RESIDING IN FRANCE}

\begin{abstract}
This article aims to analyze the construction of family space in the elderly born in North and West Africa and residing in France, far from their nuclear family living in the country of origin. It seeks to understand how this construction takes place along the individual and family trajectory and results from both constraints and spatial strategies. He develops the history of this migration through the constructed status of "geographical single" and shows how this impacts the family in its spatial configuration, organization and relationships to space.
\end{abstract}

KEYWORDS: Geography, family, migration, trajectory, celibacy

Recibido: 08-06-2018/Aceptado: 09-11-2018 


\section{Introduction}

Les migrations nord-ouest-africaines en France sont historiquement des migrations «sans famille $»^{1}$. Les individus, des hommes pour l'essentiel, ayant migré seuls, pour des raisons d'ordres différents : politiques migratoires des pays d'immigration dictées par des besoins ${ }^{2}$ notamment militaires (soldats) et économiques (travailleurs), restrictives quant au regroupement familial ; contexte politique, social et économique des pays d'émigration, incitant au départ ; rôles sociaux culturels de l'homme l'assignant à aller chercher un travail et subvenir aux besoins de la famille ; projet migratoire incluant une perspective de retour plus ou moins rapide.

La vie en famille dans ses rapports, dans sa configuration et dans son espace est alors compliquée par les aspects cités qui marquent la trajectoire de ces migrants jusqu'à la vieillesse, pour nombre d'entre eux.

On s'intéresse ici aux retraités nord-ouest-africains résidant en France dont les conjointes et les enfants sont restés dans le pays d'origine. Quand les conjointes et/ou les enfants rejoignent ces retraités à l'âge de la vieillesse, ils et elles rentrent également dans notre population-cible. L'étude des parcours et du vécu de cette migration d'hommes seuls dont certains ont été recrutés dans le pays d'origine ${ }^{3}$ et installés en France, en tant que travailleurs mais aussi en tant que conjoints et/ou pères, révèle une histoire singulière, marquée par l'évolution des conditions de leur présence en France.

Cet article prend place à la croisée de différentes dimensions de la géographie. La géographie sociale ${ }^{4}$ qui « confronte l'individu à l'épreuve de l'espace $»^{5}$ et montre l'interaction entre le spatial et le social. La géographie des migrations ${ }^{6}$ qui à travers les notions de

\footnotetext{
${ }^{1}$ SAYAD, Abdelmalek : «Le foyer des sans-famille », Actes de la recherche en sciences sociales, 32, 33 (avril/juin 1980), 89-103 / Paternalisme et maternage.

${ }^{2}$ L'histoire des migrations en France et en Europe, contexte dans lequel prend place la migration nord-ouest africaine en France, montre que les flux migratoires varient principalement en fonction des «booms » et des récessions économiques entre recrutement de travailleurs migrants et expulsion/déportation du plus grand nombre d'entre eux. Ce sont souvent les statuts précaires assignés à ces migrant-e-s qui permettent le passage de l'un à l'autre.

3 Pour rappel, l'immigration de travail organisée et revendiquée comme telle par les autorités françaises marque officiellement un arrêt en 1974. Elle reprendra sous d'autres formes selon la conjoncture économique et la nature des besoins.

${ }^{4}$ RAFFESTIN, Claude : «Territorialité : Concept ou Paradigme de la géographie sociale ? », Geographica Helvetica, 41, 2 (juin 1986), 91-96.

DI MEO, Guy : Introduction à la géographie sociale, Paris, Armand Colin, 2014, 191 p.

${ }^{5}$ LUSSAULT, Michel : « Compétences de spatialité », EspacesTemps.net, (juillet 2014).

6 JOLIVET, Violaine : «La notion de trajectoire en géographie, une clé pour analyser les mobilités ? ", EchoGéo, 2 (septembre / novembre 2007), 11 p.

SIMON, Gildas : La planète migratoire dans la mondialisation, Paris, Armand Colin, 2008, 255 p.

IMBERT, Christophe, DUBUCS, Hadrien, DUREAU, Françoise, GIROUD, Matthieu : D'une métropole à l'autre. Pratiques urbaines et circulations dans l'espace européen, Paris, Armand Colin, 2014, 485 p.
} 
migration, de mobilité et de circulation souligne les enjeux et limites de définition et de catégorisation. La géographie du droit $^{7}$ qui questionne les rapports entre le spatial et le juridique. Elle permet d'examiner les stratégies spatiales au regard de contraintes relatives aux droits $^{8}$. La géographie du vieillissement qui problématise le vieillissement à travers l'espace de vie?

Cette contribution s'appuie également sur les travaux portant sur le vieillissement en migration en France ${ }^{10}$. Elle s'inscrit enfin dans le sillage des études sur la famille dans un contexte migratoire ${ }^{11}$ et genré ${ }^{12}$.

\footnotetext{
7 BLOMLEY, Nicholas: "Geography of law", in Johnston Ronald John, Gregory Derek, Pratt Geraldine, Watts Michael (dir), The dictionary of Human geography, Somerset, Wiley-blackwell, 2000, 435-438.

FREEMAN Michael: "Law and geography : only connect ?", in Holder Jane, Harrison Carolyn (dir), Law and géography, oxford, Oxford University Press, 2002, 169- 189.

DELANEY David : "Le juridique, le spatial et la pragmatique de la construction de la réalité", in Forest Patrick (dir), La géograpbie du droit. Épistémologie, développement et perspectives, Québec, Les presses de l'université Laval, 2009, 117-135

FOREST Patrick (dir) : Géographie du droit. Épistémologie, développement et perspectives, Québec, Les presses de l'Université Laval, 2009, 286 p.

FOREST Patrick : "Vers une géographie du droit ? Pistes de réflexion", Développement durable et territoires, 6, 1 (mars 2015), $7 \mathrm{p}$.

${ }^{8}$ BERGEON Céline : Initiatives et stratégies spatiales : le projet circulatoire face aux politiques publiques. L'exemple des Roms et des Voyageurs du Poitou-Charentes (France) et de la Wallonie (Belgique), Thèse de doctorat en géographie, non publiée. Patrick Gonin (dir), Poitier, Université de Poitier, 2011, 488 p.

${ }^{9}$ MEMBRADO Monique, ROUYER Alice (dir) : Habiter et vieillir. Vers de nowvelles demeures, Toulouse, ERES Pratiques du champ social, 2013, $280 \mathrm{p}$.

${ }^{10}$ GUICHARD Eric, LECHIEN Marie-Hélène, NOIRIEL Gérard (dir) : Le vieillissement des immigrés en région parisienne, Paris, Fonds d'action sociale pour les travailleurs immigrés et leurs familles, 1992, 212 p.

SAYAD Abdelmalek : La double absence, des illusions de l'émigré aux souffrances de l'immigré, Paris, Du Seuil, 1999, $448 \mathrm{p}$.

JOVELIN Emmanuel : "Le dilemme des migrants âgés. Entre le désir du retour et la contrainte d'une vie en France", Pensée plurielle, 6, 2, 2003, 109-117.

ATTIAS-DONFUT Claudine : L'enracinement. Enquête sur le vieillissement des immigrés en France, Paris, Armand Colin, 2006, $358 \mathrm{p}$.

BERNARDOT Marc : Le grand âge en foyer de migrants : nouvelles figures, nowveaux enjeux, Bordeaux, 2ème Congrès de L'Association Française de Sociologie, 2006, 9 p.

CHOUKRI Hmed : "Hors thème. Les immigrés vieillissant en foyer pour travailleurs ou les habitants de nulle part", Retraite et société, 47, 1, 2006, 137-159.

EMSELLEM Sylvie : "Décrypter le vieillissement des immigrés par le prisme de leurs liens familiaux", L'Année du Maghreb, 3, 2007, 601-614.

BERNARDOT Marc: Loger les immigrés, la Sonacotra 1956 - 2006, Broissieux, Editions Du Croquant, 2008, $296 \mathrm{p}$.

MATH Antoine : "L'accès des vieux migrants aux droits sociaux : un chemin semé d'embûches", Collectif des Accidentés du Travail, Handicapés et Retraités pour l'Égalité des Droits, 2009.

HOST Jérôme, PEPIN Juliette : "Justice et dignité pour les chibanis", Plein droit, 93, 2, 2012, 8-11.

JAEGER Marcel, MADOUI Mohamed : "Les migrants face aux défis du vieillissement", Hommes et migrations, 1309, 2015, 6-7.

${ }^{11}$ AMBROSINI Maurizio : «Séparées et réunies : familles migrantes et liens transnationaux ». Revue Européenne des Migrations Internationales, 24, 3, 2008, 79-106.

ATTIAS-DONFUT Claudine, WOLFF François-Charles : Le destin des enfants d'immigrés : Un désenchainement des générations, Paris, Stock, 2009, 306 p.

${ }^{12}$ OSO CASAS Laura : «Migration, genre et foyers transnationaux : un état de la bibliographie », Les cahiers $d u$ CEDREF, 16, 2008, 125-146.
} 
Les migrants nord-ouest-africains, vieillissants en France et dont la famille est restée dans le pays d'origine, représentent une population hétérogène et les trajectoires ainsi que les configurations familiales les concernant sont diverses. Dans cette hétérogénéité et dans cette diversité de catégories, de cas et de situations, la notion de contrainte ressort comme constante. Le vécu et la construction de l'espace familial sont marqués par cette notion de contrainte qui a des retombées sur les individus et la famille et les pousse à une réadaptation permanente.

Cette problématique nous amène à interroger la géographie de la famille en situation de contraintes matérielles et/ou subjectives. L'objectif ici est de proposer quelques pistes pour saisir la construction de l'espace familial à partir d'un contexte migratoire déterminé par des aspects politiques, sociaux et spatiaux. Comment se construit l'espace familial ? Avec quels limites et enjeux, compte tenu de l'éclatement familial et des contraintes pesant sur les mobilités?

Dans un mode de vie où les circulations et les relations sont cadrées par les politiques migratoires et où les trajectoires professionnelles et les statuts - tel que celui de travailleur immigré célibataire géographique - impactent les trajectoires individuelles et familiales, comment s'organise la famille et qu'est-ce que la contrainte au célibat géographique ?

Ces questionnements concernent également les enfants, d'une part, à travers le fait qu'ils et elles aient grandi dans une sorte de famille monoparentale et, d'autre part, à travers les événements que constituent l'arrivée du père à la retraite, la dégradation de sa santé, la venue de la mère en France ou encore leur propre implication dans la migration internationale.

Enfin, quelles sont les stratégies de contournement utilisées à un niveau individuel ou familial ? Et quel genre de vivre en famille produisent-elles?

On s'intéresse ici au statut administratif, spatial et social de travailleur immigré célibataire géographique en France, en partant de l'idée que ce statut place la famille dans une situation de contrainte. Il faut donc travailler sur les aspects géographiques, économiques, politiques, sociaux et culturels de ces contraintes.

S'agissant de notre terrain d'enquête, nous avons commencé à le constituer en 2014 à Montpellier ${ }^{13}$, en assurant, avec l'Association des Travailleurs Maghrébins de France (ATMF), des permanences d'accès aux droits sociaux sanitaires à destination des personnes âgées

${ }^{13}$ Cette enquête a servi à la réalisation d'un mémoire de master portant sur cette question de la construction de l'espace familial : ALAHYANE, Yacine : Trajectoire et (im)mobilités dans les circulations internationales : Regard sur la construction de l'espace familial. Le cas des Nord-Africain-e-s âgé-e-s et isolé-e-s vivant à Montpellier, Mémoire de Master Développement Durable et Aménagement du territoire, Geneviève Cortès (dir.), Montpellier, Université Montpellier 3, 2015, 249 p. 
immigrées. Ces permanences ont eu lieu dans les locaux du Centre Social Caisse d'Allocation Familiale du quartier de La Mosson et dans la Librairie-Bibliothèque Scrupule. Elles ont consisté à écouter, informer, orienter et accompagner individuellement dans les démarches administratives des migrant-e-s vieillissant-e-s ayant une demande socio-sanitaire. L'orientation du public vers ces permanences a été faite, pour certaines personnes, par le Centre Social. Nous en avons rencontré d'autres dans les espaces publics et dans les Foyers de Travailleurs Migrants de la ville où nous avons diffusé des informations relatives à ces permanences. Ainsi, nous avons interagi avec une trentaine de personnes dont une dizaine sur une longue durée (entre 4 et 24 mois).

Ces permanences d'accès aux droits constituent un espace et un temps d'interaction avec les migrant-e-s vieillissant-e-s enquêté-e-s et avec leurs familles. Les échanges portent sur le vécu et la trajectoire des personnes de façon globale et également sur des problèmes administratifs à résoudre (demande de retraite, de couverture médicale, de prise en charge de la perte d'autonomie, de regroupement familial, etc.) ainsi que sur des questions de droit en général. Dans le cadre de ces permanences, nous sommes également en interaction avec des intervenants du socio-sanitaire (agents des services sociaux, des caisses de retraite, et d'autres administrations) à travers des échanges par téléphone, mail, courrier.

En plus des données récoltées dans le cadre physique des permanences, nous avons effectué des déplacements avec les personnes âgées, notamment auprès de diverses administrations (Mutualité sociale agricole, Caisse d'Allocations Familiales, Pôle emploi, Caisse Primaire d'Assurance Maladie), ou auprès d'autres professionnels (avocats, associatifs, etc.) ou encore chez elles.

Ainsi, ces permanences menées à Montpellier nous ont permis de réunir un corpus composé de différents types de données : discours oraux (nos échanges avec le publiccible, nos échanges avec les institutionnels, interactions entre le public-cible et des agents administratifs, etc.), discours écrits et littérature grise (courriers administratifs, codes et textes législatifs, différents formulaires de demande d'ouverture de droits, etc.) ; observations de lieux, de parcours, de procédures en lien avec les pratiques de l'accès aux droits.

Parmi les personnes rencontrées dans le cadre de ces permanences, nous avons pu mener un entretien semi-directif individuel avec quatre d'entre elles et un entretien semidirectif collectif avec une famille constituée d'une mère et de ces deux filles. Le choix de ces personnes tient au fait qu'elles correspondent, d'une part, au profil du travailleur immigré/retraité célibataire géographique, ciblé par notre problématique de recherche et 
d'autre part, au profil de l'épouse et celui des enfants de travailleur immigré/retraité, venue-s s'installer en France avec ou sans le mari/père.

Depuis 2016, dans le cadre d'une thèse ${ }^{14}$ en Cifre ${ }^{15}$, nous poursuivons cette recherche, au sein de l'association Groupe de Recherche pour le Développement Rural (Grdr) qui mène des programmes d'accès aux droits et de lutte contre l'isolement, à destination des migrant-e-s vieillissant-e-s.

Montreuil, Paris $19^{\mathrm{e}}$ et plus particulièrement Aubervilliers constituent notre terrain d'enquête actuel. Nous y assurons le même type de permanences d'accès aux droits et d'accompagnements dans les démarches administratives et nous y collectons le même type de données décrites ci-dessus. S'ajoute à cela, l'accès aux archives de l'association où figurent de nombreux dossiers de suivi en lien avec les permanences.

Par ailleurs, notre terrain d'enquête est enrichi par d'autres activités de recherche. Il s'agit de réunions d'information collectives sur les thèmes relatifs à la retraite et à la santé. Ces temps d'échanges collectifs sont co-animés par le Grdr et des professionnels tels que ceux du CICAS $93^{16}$ ou ceux de la Direction de la Santé Publique et de l'Atelier Santé Ville d'Aubervilliers. Ils visent tantôt les personnes âgées immigrées, tantôt des intervenant-e-s du secteur social et médico-social. Il s'agit également de réunions partenariales tenues dans le cadre des programmes du Grdr, portant sur l'accès aux droits des personnes âgées immigrées et de la mise en œuvre de ces programmes. Il s'agit aussi de rencontres/forums/journées d'étude ou de formations, organisés par le Grdr ou par d'autres structures (municipalités, départements, associations, etc.) et, portant sur les questions d'accès aux droits, de migration, de vieillissement, de dématérialisation des services publics, etc.

Ces activités nous permettent de réunir des données diverses : discours oraux (présentations/animations/interventions faites par des institutionnels devant différents publics, nos échanges avec ces institutionnels, interactions entre différents intervenant-e-s du secteur socio-sanitaire, etc.), discours écrits (brochures, rapports et autres livrables); observations de lieux, de pratiques organisationnelles, de communications.

Dans le cadre des permanences d'accès aux droits, des accompagnements et des réunions d'information collectives, menés avec le Grdr, nous avons interagi avec plus d'une centaine de personnes nées dans un pays n'appartenant pas à l'Union Européenne et

\footnotetext{
${ }^{14}$ Thèse en cours portant sur : Le vieillissement en migration à l'épreuve du territoire et de l'accès aux droits : le vécu de personnes âgées, nées en Afrique du Nord-Ouest, résidant à Aubervilliers, sous la direction de Geneviève Cortes et d'Isabelle Berry-Chikhaoui, laboratoire Art-Dev, UMR 5281, Université Montpellier 3.

${ }^{15}$ Convention Industrielle de Formation par la Recherche.

${ }^{16}$ Centre d'Information de Conseil et d'Accueil des Salariés Seine-Saint-Denis.
} 
vieillissant en Ile-De-France. Dans ce cadre-là, nous avons eu des échanges réguliers sur plusieurs mois (entre 6 et 24 mois) avec une trentaine d'entre elles, nées en Afrique du NordOuest. Nous avons également mené un entretien semi-directif individuel avec 26 personnes âgées nées en Afrique du Nord-Ouest résidant soit à Aubervilliers, soit à Montreuil ou à Paris. Une vingtaine d'entre elles venaient aux permanences. Ajoutons à cela une série de deux focus groups, organisés à Aubervilliers avec une dizaine de personnes et portant sur le vécu de l'accès aux droits en situation de vieillissement, de migration et de mutation des politiques publiques (dématérialisation, fermeture des guichets, multiplication des contrôles, etc.). Précisons ici que toutes les personnes ayant participé à ces focus groups ont été rencontrées dans le cadre des permanences et des réunions d'information collectives et ont fait un entretien semi-directif.

Ainsi, notre terrain d'étude, présenté ici, nous amène à enquêter - outre les acteurs et les intervenants du domaine social, socio-sanitaire et administratif - auprès d'une large catégorie, celle des migrant-e-s vieillissant-e-s en France d'une maniéré générale et, celle des personnes nées en Afrique du Nord-Ouest en particulier. Si ces catégories ne s’inscrivent pas toutes dans le célibat géographique, notamment au moment de la vieillesse ${ }^{17}$, nous considérons que la prise en compte de leur trajectoire et de leur vécu permet un regard croisé sur les questions soulevées ici.

Pour tenter de répondre à ces questions, on reviendra d'abord, à travers la construction d'un célibat géographique, sur le contexte de la migration de la population-cible afin d'éclairer sa situation. On se focalisera ensuite sur la construction de l'espace familial, sur son articulation avec un ensemble de dimensions notamment politiques, sociales, économiques, sanitaires et sur ses enjeux.

\section{La construction d'un célibat géographique}

En retraçant les étapes-clefs de la trajectoire du travailleur immigré, de son installation en France jusqu'à son vieillissement, l'objectif ici est d'interroger le processus qui conduit à l'inscrire dans le célibat géographique. Cette notion renvoie souvent aux migrants n'ayant pas fait ou pas pu faire de regroupement familial ${ }^{18}$. De façon plus globale, elle est

\footnotetext{
17 BERNARDOT, Marc : 2006, op. cit. ; ATTIAS-DONFUT, Claudine : 2006, op.cit. ; BAROU, Jacques : « Vieillir en terre étrangère : trois catégories d’immigrés en France », Ethnologie française, 3, 171 (2018), 479-488. MARTINEAU, Aurélien, PLARD, Mathilde : «Les personnes âgées immigrées à l'épreuve du SuccessfulAging », Cybergeo : European Journal of Geography, Politique, Culture, Représentations, document 853, (mis en ligne le 03 mai 2018).

${ }^{18}$ GALLOU, Rémi : «Les immigrés isolés : la spécificité des résidents en foyer », Retraite et société, 1, 44 (2005),
} 
utilisée en sciences humaines et sociales pour l'étude des mobilités professionnelles dans leurs interactions avec la sphère familiale et la distance géographique ${ }^{19}$. Constatons enfin que cette notion est passée dans le langage courant puisque qu'une simple recherche sur internet nous oriente vers des forums, des blogs et des revues grand public qui l'utilisent.

\subsection{La migration d'hommes seuls}

Entre 1950 et 1974, pour répondre notamment à un double objectif économique et démographique, l'état français a eu recours à une immigration massive. Les flux de cette migration de travail d'après-guerre se poursuivront avec des phases de répit ou d'accélération jusqu'en 1980. Durant une trentaine d'années, la flexibilité de la gestion de ces flux migratoires constituera pour l'Etat et le patronat français le principal moyen de réguler les aléas de la conjoncture. La population d'origine « extérieure » restera ainsi vouée à la régulation des besoins en main-d'œuvre ${ }^{20}$.

Certains travaux tels que ceux de l'Association des Mineurs Marocains du NordPas-de-Calais, de Saïd Bouamama et de Jessy Cormon ${ }^{21}$, de Marc Bernardot ${ }^{22}$, ainsi que le film documentaire de Yamina Benguigui, « Mémoires d'immigrés, l'héritage maghrébin ${ }^{23}$, montrent que la migration étudiée ici et ses modalités ont été fixées par un cadre politique, juridique et administratif. Ce cadre est déterminé et caractérisé notamment par la mise en place d'institutions telles que l'Office National de l'Immigration (ONI), créé en 1945, qui a le monopole des opérations de recherche, de sélection et d'acheminement de ce qui est désigné comme « travailleurs étrangers en France ». Citons également la Société Nationale de Construction pour Travailleurs d'Algérie (SONACOTRA) qui voit le jour en $1956^{24}$ et qui construit et gère ce qui institutionnellement deviendra et s'appellera des

106-147. ATTIAS-DONFUT, Claudine: 2006, op. cit.

19 VIGNAL, Cécile, « Devenir 'célibataire géographique' ? Arbitrages conjugaux et familiaux suite à la délocalisation d'une usine », Cabiers du Genre, 2, 41 (2006),139-157.

${ }^{20}$ CONUS, Marie-France, ESUDIER, Jean-Louis : « Analyse économique du cycle de vie de l'industrie houillère française : la phase ultime (1970-2004) », Séminaire G.D.R. Energie, Paris, 2004, 23 p.

${ }^{21}$ ASSOCIATION DES MINEURS MAROCAINS DU NORD-PAS-DE-CALAIS (AMMN): Du bled aux corons, un rêve trabi, Dechy, AMMN, 2008. À consulter ou acheter à l'Association de Mineurs Marocains du Nord (AMMN), 33, rue Casimir-Beugnet, 59187 Dechy.

${ }^{22}$ BERNARDOT, Marc: 2008, op. cit.

${ }^{23}$ BENGUIGUI, Yamina: « Mémoires d'immigrés, l'héritage maghrébin », YouTube, 2h32min, URL : https://www.youtube.com/watch?v=mXbmjmO5rX8, Consulté le 30/05/2018.

${ }^{24}$ La Société Nationale de Construction pour Travailleurs d'Algérie créée en 1956 construit et gère des foyers de travailleurs migrants. La Sonacotra, sur critère ethnique/national, « offre » d'abord aux Algériens avant de s'élargir à l'ensemble des migrants - (post)coloniaux en particulier - des conditions de « résidence temporaire et en-dessous des normes de logement pour des actifs seuls» (M. Bernardot, 2008, 15). La Sonacotra qui a un statut juridique hybride de société d'économie mixte nationale combinant la tutelle de plusieurs ministères, celui de l'intérieur notamment, et des acteurs privés au sein du capital, constitue un exemple de traitement de 
«Foyers de Travailleurs Migrants ».

Il est important de noter que ces deux exemples d'institutions, qui renvoient l'une au recrutement, et donc au travail, et l'autre, au logement, représentent un système qui, comme le décrit Sayad ${ }^{25}$, lie, dans une relation interdépendante, le logement et le travail. Ainsi ce système a défini le statut de l'immigré et son « « existence » (officielle) » ${ }^{26}$.

Ce système montre, selon nous, que ce qui est ciblé ici, c'est l'homme seul. En Afrique du Nord-Ouest, l'ONI recrute principalement des hommes seuls et 《l'introduction ${ }^{27}$ des familles en France n'est pas sa priorité. Même après les indépendances, les recrutements d'hommes seuls se poursuivent par le biais d'accords migratoires négociés uniquement en termes de migration de travail. La SONACOTRA, quant à elle, loge des hommes seuls.

Le statut de séjour en France, quant à lui, autre maillon du système, est précaire et, l'homme seul est administrativement subordonné au travail (et au logement). Ainsi, stabiliser sa situation administrative et obtenir la carte de résidence longue durée, relève du parcours du combattant. Rappelons ici que l'ONI recrutait avec un système de contrats temporaires et renouvelables, ce qui en termes de droit du salarié, renvoie, à l'époque, à ce qu'il y a de plus précaire et de plus flexible - à côté du statut de travailleur sans-papiers qui commence à être de plus en plus présent - Le titre de séjour, quant à lui, correspond à la durée du contrat.

Ainsi, ces institutions ont participé à la construction du statut, du fait et de l'image de «travailleur immigré/homme seul». Cette figure se retrouve à la croisée de différentes représentations socioculturelles et « catégories par lesquelles on définit l'immigré et par lesquelles on délimite son espace social » ${ }^{28}$, révélant « l'idée que l'on se fait de l'immigré et qui contribue à faire l'immigré » ${ }^{29}$. Nous avons déjà mentionné la représentation qui impose à l'homme le rôle de pourvoyeur d'argent pour la famille. Ajoutons celle de l'immigré venant d'un « pays pauvre » dans un « pays riche », un " pays d'accueil » où il trouvera de quoi subvenir aux besoins des siens et même constituer un capital dans son pays d'origine.

La problématique des liens entre mobilité, travail, genre et famille est posée par ces

la population ouvrière par le biais du logement social qui relie l'action policière dans le contexte de la guerre en Algérie et de besoin de main-d'œuvre docile. Le 23 janvier 2007, la Sonacotra change de nom et devient Adoma, nom construit à partir du latin « ad » qui signifie vers et « domus », la maison. Tout un symbole ! ${ }^{25}$ SAYAD, Abdelmalek : (avril/juin 1980), op. cit.

${ }^{26}$ Ibidem.

27 Terme utilisé par les institutions étatiques dans des circulaires telles que celle du 20 janvier 1947 ou encore sur le site internet actuel de l'Office Français de l'Immigration et de l'Intégration (ancienne

ONI) : http://www.ofii.fr/qui-sommes-nous/notre-histoire

${ }^{28}$ SAYAD, Abdelmalek : (avril/juin 1980), op. cit.

${ }^{29}$ Ibidem 
modèles. Le modèle patriarcal des Trente Glorieuses basé sur l'image du «travailleur » et de la « ménagère $»^{30}$ implique une mobilité pendulaire du travailleur entre son lieu de travail et son foyer où vit la « ménagère $»^{31}$. Le second modèle, quant à lui, repose sur l'image d'un « travailleur » qui ne retourne auprès de la « ménagère » que durant le congé annuel, ou lors d'un retour définitif, à la retraite ou une fois un capital constitué $e^{32}$.

Ainsi, l'homme, jeune travailleur immigré, au début de son projet migratoire, rentre dans un processus qui peut le placer entre lieu de travail et de revenus d'une part et, lieu de famille et de projet d'avenir, d'autre part. Il s'agit aussi d'un processus où l'administration et le travail peuvent fixer les retrouvailles avec cette famille restée au pays ${ }^{33}$.

A ce stade de la migration, la famille renvoie aux parents, à la fratrie, à la famille élargie et à la communauté. Pour certains, elle renvoie aussi à des conjointes et éventuellement des enfants. Relevons cependant que nombre d'hommes rencontrés au cours de nos recherches se sont mariés dans leur pays d'origine, dans les années qui ont suivi leur installation en France. Quoiqu'il en soit, cette première phase qui correspond à celle du départ, de l'installation et des premiers va-et-vient, se fait, notamment pour la famille, pour améliorer ses conditions de vie ou pour la fonder.

Cependant, les réalités de cette migration (salaire d'ouvrier, conditions socio-économiques du pays d'origine) compromettent le projet de retour. Les conditions de vie de la famille dépendent des revenus du travailleur immigré qui de son côté rentre dans un processus d'enracinement - administratif, social, économique et professionnel, etc.- en France ${ }^{34}$. L'immigré et sa famille s'installent ainsi dans un provisoire qui dure ${ }^{35}$ et dans un mode de vie qui matérialise la construction d'un célibat géographique.

Ainsi, l'immigration des années 1950-1974 a été marquée par une surreprésentation masculine et par ce célibat géographique ${ }^{36}$. C'est particulièrement le cas des Nord-Ouest-

\footnotetext{
${ }^{30}$ Nous désignons par là des catégories.

31 Sur l'évolution actuelle de ce modèle et sur le poids de ces assignations sociales à des rôles sexués, voir la revue : Cabiers du Genre, 2, 41 (2006), 220 p. Ou encore les travaux de Nathalie Ortar : ORTAR, Nathalie, " Qui bouge, qui reste dans le couple ?», e-Migrinter, 11 (2013), 94-102.

32 SAYAD, Abdelmalek, BOLZMAN, Claudio, FIBBI, Rosita, GUILLON, Michelle : La vacance comme pathologie de la condition d'immigré. Le cas de la retraite et de la pré-retraite », Revue européenne des migrations internationales, 17, 1 (2001), 11-36 / Émigrés-Immigrés : vieillir ici et là-bas.

CARTILLIER, Jeanne : Vieillesses nomades. Les migrants maghrébins de la première génération dans le va-et-vient, Séminaire « Habitat et Société », BAROU, Jacques(dir.), HOFMANN, Bernard (dir.), Université Pierre Mendes France, Institut D'études Politiques de Grenoble, IEP (éditeur), 2002, 150 p. BAROU, Jacques : (2018), op. cit.

MARTINEAU, Aurélien, PLARD, Mathilde : (mis en ligne le 03 mai 2018), op. cit.

33 ALAHYANE, Yacine : 2015, op. cit.

${ }^{34}$ Ibidem

35 ASSOCIATION DES MINEURS MAROCAINS DU NORD-PAS-DE-CALAIS (AMMN): 2008, op. cit.

${ }^{36}$ MADOUI, Mohamed : «Le vieillissement des migrants à l'épreuve des sciences sociales », Vie sociale, 4, 16 (2016),15-28.
} 
Africains - pour qui ont été initialement construits les foyers de travailleurs migrants - qui constituent encore, actuellement, la majorité des habitants de ces foyers ${ }^{37}$.

\subsection{Le (non)regroupement familial}

Au cours des années 1970, les crises du capitalisme marquent un changement dans les besoins et donc dans les politiques migratoires. La migration de travail d'individus seuls change elle aussi. Elle devient encore plus précaire, sans-papiers. La migration au titre du regroupement familial constitue alors l'un des principaux modes d'installation légale en France. Beaucoup arrivent à faire venir leurs familles en France.

On peut dire ici que, pour le travailleur immigré, faire venir sa famille en France correspond à des stratégies migratoires ${ }^{38}$ et peut s'expliquer par différents facteurs.

Remédier aux inconvénients de l'éclatement familial - affectifs, culturels, socioéconomiques, logistiques dans un contexte de répartition genrée des tâches - en constitue l'un des principaux. Pour le travailleur immigré, faire venir près de lui conjointe et enfants, c'est aussi miser sur les possibilités offertes par le «pays d'accueil », le « pays riche »: amélioration des conditions matérielles, aides sociales à la famille, scolarisation des enfants, ascension sociale, etc.

Quoiqu'il en soit, d'autres travailleurs immigrés resteront célibataires géographiques, dans le sens où ils vivent et travaillent en France, tandis que leurs conjointes et leurs enfants vivent dans le pays d'origine. Cette situation se prolongera jusqu'à la vieillesse. Nous avons pu constater que pour certains, le regroupement familial n'a pas été fait, en raison de stratégies personnelles, familiales ou encore communautaires ${ }^{39}$.

Ces stratégies laissent entrevoir, notamment, une volonté de diversification des revenus à un niveau transnational ou un désir de ne pas tout laisser tomber dans le pays d'origine. Ainsi, comme en témoignent plusieurs de nos enquêtés, la famille nucléaire qui reste, est gardienne de terres agricoles, d'oliviers, de têtes de bétail, de commerces, etc. Il y a aussi les raisons affectives liées à la présence d'un parent qu'on ne peut pas laisser seul sur le territoire de départ. La famille nucléaire joue donc ce rôle de prendre soin du parent.

\footnotetext{
37 ATSE N’CHO, Jean-Baptiste : «Les foyers de travailleurs migrants en région parisienne : "copies" des villages africains ou centres de mutations sociopolitiques? ", Influxus, (mis en ligne le 11 septembre 2014), Consulté le 1er novembre 2018.

38 BOUMAZA, Nadir : «Logement, habitat et fixation des Maghrébins de France », Espace, populations, sociétés, 2, 3 (1996), 315-323 / Immigrés et enfants d'immigrés.

WIHTOL DE WENDEN, Catherine : «L'intégration en France à la lumière de deux rapports récents du Haut Conseil à l’intégration », Hommes \& migrations, 1294 (2011), 18-23.

${ }^{39}$ Ce constat rejoint les résultats de l'étude : BOUMAZA, Nadir : (1996), op. cit.
} 
Toutefois, le regroupement familial est sévèrement conditionné. Il n'a été institutionnalisé qu'en 1976, dans un contexte de crise politique, sociale, économique. La législation française en a restreint plus d'une fois les critères : surface de logement requise en fonction du nombre de membres attendus, montant des revenus et stabilité du travail, liens familiaux limités à la famille nucléaire, âge des enfants, durée du séjour en France, sincérité de l'engagement matrimonial, stabilité du travail.

Répétons les propos de Monsieur O., ancien ouvrier agricole, qui en passant de patron en patron, s'est «fatigué » à essayer de ramener sa femme et ses enfants, sans résultat, selon lui, faute « de travail stable », de CDI :

«Je n'ai pas pu le faire, je n'arrivais pas à trouver de travail stable. Quand je travaillais 2 ou 3 mois chez un patron, que je sentais que c'était une personne bien, je lui demandais : «Monsieur, faites-moi plaisir, je voudrais ramener ma famille ». Il me répondait : «Non, non, non, non, non ! » Et pour les patrons suivants, c'était la même chose. Je voulais faire le regroupement familial mais ce n'était pas possible. Au bout d'un moment je me suis fatigué et j'en ai eu assez ».

Ainsi, nous avons pu observer que beaucoup de personnes n'ont pas pu mener jusqu'au bout cette procédure, faute de travail stable, d'entrée d'argent suffisante et/ou de logement convenable. Tout au long de leur parcours, le statut de célibatairisé marque leurs rapports à l'administration, à l'espace et aux (im)mobilités dans leurs modes d'organisation contraints et entre « ici » et « là-bas ».

\subsection{Le retraité célibataire géographique}

Aujourd'hui, parmi ces populations, une catégorie de personnes parvient à l'âge de la retraite, voire au stade de la fin de vie. Pour le travailleur célibataire géographique, cette étape de la retraite et du vieillissement repose la question du retour et de l'espace familial. En effet, le travail, qui, jusque-là, justifiait tant sa présence en France, prend fin. On peut observer que certaines personnes rentrent définitivement dans le pays d'origine ${ }^{40}$, que d'autres, minoritaires, restent en France, ne rentrent plus au pays et, s'inscrivent dans un processus de

${ }^{40}$ MADOUI, Mohamed : (2016), op. cit. 
rupture, voulue ou contrainte, plus ou moins totale avec la famille vivant dans le pays d'origine $^{41}$. Beaucoup font des allers-retours ${ }^{42}$.

A cette étape, notons également que l'immigré vieillissant peut être rejoint par sa conjointe, dans le cadre d'une procédure de visa ou de regroupement familial, toujours conditionnés. Il arrive aussi que les enfants, maintenant adultes, viennent le rejoindre, souvent sans papiers.

Nous avons constaté que la venue en France de la conjointe est parfois perçue de façon péjorative, notamment par des intervenant-e-s du domaine social : «elle vient uniquement à la demande du mari vieillissant et malade pour s'occuper de lui ». Toutefois, cette perception réductrice ne tient pas compte, d'une part, des restrictions administratives et temporelles du regroupement familial. En effet, il est plus facile de faire venir la conjointe seule, donc à la vieillesse, les enfants étant devenus autonomes, que la conjointe avec les enfants, lorsque ces derniers sont encore en bas-âge. D'autre part, cette perception ne prend pas en considération les stratégies familiales visant à faciliter la circulation de la conjointe, sa protection sociale, l'accès à ses droits à la pension de réversion et au minimum vieillesse que pourraient lui garantir le regroupement familial et le statut administratif de « résidente » en France.

Rester en France après le passage à la retraite peut s'expliquer par un attachement au lieu de vie, de résidence, de sociabilité et donc à des habitudes et à un mode de vie ${ }^{43}$. Cette situation est aussi en lien avec des réalités politiques, administratives, sociales, économiques et sanitaires, qui contribuent à prolonger dans la vieillesse, la situation de célibat géographique.

De nombreux rapports et études ${ }^{44}$ traitant de la condition sociale, sanitaire et économique actuelle des immigré-e-s âgé-e-s montrent un passage à la vieillesse qui est jugé plus inquiétant que pour la plupart des individus. Nos observations de terrain vont dans ce sens et nous ont permis de constater une situation marquée notamment par :

a) Un vieillissement prématuré en lien notamment avec les mauvaises conditions de travail et de logement. Nous avons également remarqué que les personnes vivant sans la famille ont tendance à avoir un mode de vie marqué par la surconsommation de tabac

\footnotetext{
${ }^{41}$ BERNARDOT Marc, BOLZMAN Claudio, FIBBI Rosita, GUILLON Michelle : « Le vieux, le fou et l'Autre, qui habite encore dans les foyers ? », Revue européenne des migrations internationales,17,1 (2001), 151-164 /Émigrés-Immigrés : vieillir ici et là-bas.

42SCHAEFFER, Fanny : " Mythe du retour et réalité de l'entre-deux. La retraite en France, ou au Maroc ? ", Revue européenne des migrations internationales, 17,1 (2001), 165-176 / Émigrés-Immigrés : vieillir ici et là-bas. JAEGER, Marcel, MADOUI, Mohamed : (2015), op. cit.

${ }^{43}$ SCHAEFFER, Fanny : (2001), op. cit.

ATTIAS-DONFUT Claudine : 2006, op. cit.

${ }^{44}$ Cités ci-dessus.
} 
et les mauvaises habitudes alimentaires. L'isolement familial peut être à l'origine de nombreuses carences et pathologies. Nous avons enfin noté que le vieillissement, la perte d'autonomie, le suivi médical, les rendez-vous chez les spécialistes, leur fréquence et leur intensité posent problème aux besoins de mobilité internationale dans le contexte de cette migration.

b) Une précarité économique : les personnes immigrées sont surreprésentées au sein des allocataires du Minimum Vieillesse, qui porte le nom d'Allocation de Solidarité aux Personnes Agées (ASPA). Cela est dû au faible taux de cotisation qui est lui-même lié à des parcours professionnels atypiques et précarisants. L'ASPA, comme d'autres prestations sociales (Revenu de Solidarité Active, Couverture Maladie Universelle, etc.) est soumise à la condition de résidence en France. Il faut rester 6 mois et un jour par an sur le territoire français, et les caisses de sécurité sociale multiplient les contrôles auprès des vieux migrants, ciblant en particulier ceux vivant en foyer ${ }^{45}$.Si le temps passé hors de France est jugé trop long, les personnes sont considérées comme «non résidentes » et la quasi-totalité de leurs droits sociaux en matière de minimum vieillesse, d'aides au logement ou de protection maladie, leur est supprimée, souvent sans que la décision ne leur soit notifiée, ni qu'elles aient la possibilité de pouvoir s'expliquer ou contester. A la suite de ces contrôles, les caisses opèrent des redressements pour de vieux migrants dont certains ont des revenus, ceux de leur retraite contributive, inférieurs à 300 euros par mois et qui se retrouvent avec des dettes envers ces caisses, allant de 1000 à 20000 euros. On voit ici comment la condition de résidence et/ou la précarité économique prolongeant la situation de célibataire géographique de l'ancien travailleur immigré dans la retraite.

c) Des problèmes d'accès aux droits : insistons ici sur la complication des démarches administratives, sur la variabilité et la segmentation des législations (droit des étrangers, droit du travail, droit de la sécurité sociale, etc.), des prestations et du travail social, sur les politiques d'austérité et leurs liens avec cette question de l'accès aux droits (dématérialisation des services publics, fermetures de guichet, suspicions, contrôles et harcèlements des plus précaires). Par ailleurs, aux problèmes d'accès aux droits concernant les retraites, les accidents du travail et l'invalidité ou encore la couverture

\footnotetext{
${ }^{45}$ Ces contrôles pratiqués aux guichets des administrations mais aussi, à domicile, par des inspecteurs, reposent notamment sur des techniques telles que la vérification des compteurs électriques et d'eau dans les chambres des foyers, ou encore par le contrôle des passeports. Il arrive que des personnes absentes pendant la période estivale ou durant certaines fêtes religieuses soient systématiquement considérées comme ne respectant pas la condition de résidence.
} 
maladie ou l'accès aux services gérontologiques, s'ajoutent d'autres aspects. Ceux-ci sont liés au statut administratif "d'immigré étranger ", à la résidence en France et à la libre circulation entre la France et le pays d'origine. Notons également que l'immigré dont la famille vit dans le pays d'origine est ainsi soumis à une double législation et aux conventions bilatérales ${ }^{46}$. Ces aspects sont enfin relatifs au droit à la vie de famille, notamment lorsque celle-ci se trouve dans le pays d'origine, d'une part, faute de regroupement familial et en l'absence de libre circulation, d'autre part, les revenus du retraité étant soumis à la condition de résidence en France.

d) Le logement des immigré-e-s âgé-e-s est marqué «par deux aspects : celui d'un habitat de mauvaise qualité et celui d'une concentration dans certains quartiers ${ }^{47}$. Si une grande partie des personnes âgées immigrées, vivant sans leur famille nucléaire dans leur logement, sont dans le parc classique, rappelons la surreprésentation historique des personnes nées en Afrique du Nord-Ouest dans les foyers ${ }^{48}$.

e) Des rapports contraints aux territoires : en effet, se pose avec acuité, chez nombre de personnes âgées immigrées, la question de la spécificité des rapports aux territoires qui sont marqués par la contrainte. C'est d'autant plus le cas pour les personnes ayant une retraite précaire, soumises à la condition de résidence pour la perception de leurs revenus et vieillissant seules en France, leur famille nucléaire vivant dans le pays d'origine. Notons également le cas des malades qui doivent consulter régulièrement et/ou être hospitalisés en France où les soins sont, souvent, largement mieux pris en charge que dans les pays d'origine.

Ainsi, les difficultés de la vieillesse en migration en France se manifestent à travers différents aspects : statut d'immigrés, contraintes légales, complexité des procédures, entraves administratives, manque d'information, relations difficiles à la langue française écrite et parlée, configuration familiale, isolement, trajectoires migratoire et professionnelle, revenus, vieillissement et santé. Ces aspects, qui relèvent de la question de l'accès aux droits, peuvent, selon les cas de figure, être interconnectés entre eux et se cumuler tout au long de la trajectoire migratoire. Ces caractéristiques qui concernent l'ensemble des immigré-e-s âgé-e-s font cependant ressortir la spécificité de la situation des personnes célibatairisées,

\footnotetext{
${ }^{46}$ Ainsi pour le travailleur comme pour le retraité, se posent les questions de la couverture médicale de la famille dans le pays d'origine, des prestations sociales, du transfert de la retraite et des droits sociaux dans le pays d'origine.

${ }^{47}$ BERNARDOT, Marc : 2008, op. cit.

${ }^{48}$ Neuf résidants sur dix en sont originaires en 1999.
} 
anciennement travailleurs immigrés.

Notre enquête nous a permis de constater que des questions simples, comme le retour au pays d'origine à l'âge de la retraite, peuvent se révéler problématiques car conditionnées par l'administration, par le statut socioéconomique et/ou par les problèmes de santé. Ces situations soulèvent ainsi des interrogations fortes sur les rapports effectifs et affectifs aux lieux, sur l'inscription et l'identification territoriales de ces populations dont on peut supposer qu'ils ne sont pas toujours harmonieux, tant pour l'individu que pour la famille.

\section{Construire l'espace familial dans l'éloignement}

Partant de la question fondamentale de la construction de l'espace familial dans le cadre d'une migration sans la famille, nous avons dégagé ci-dessus certains aspects déterminants. Dans ce qui suit, nous allons tenter d'approfondir notre réflexion sur les enjeux de la construction de cet espace familial tout au long de la trajectoire migratoire de notre population cible.

\subsection{La famille : une question spatiale}

La migration oblige à penser la famille de façon spatialisée. Elle fait émerger des formes familiales diverses voire nouvelles ${ }^{49}$. Au cours de notre enquête auprès de notre population-cible, nous avons noté que la migration internationale du père était souvent accompagnée d'une migration interne de la famille vers les villes. Une réflexion sur ce qu'est la famille dans le territoire de départ permettrait ainsi de montrer comment l'exode rural fait passer le modèle de la famille traditionnelle élargie, correspondant à celle du village et de la tribu, vers le modèle de la famille restreinte, de type conjugal, nucléaire, celui de la ville, de la société urbaine et citadine, faisant comprendre l'impact des circulations internes au pays d'origine sur les transformations du modèle familial ${ }^{50}$.

Pour ce qui est des sociabilités dans le pays d'immigration, notons que sitôt arrivé, l'homme seul «a également besoin de se rapprocher de parents réels ou supposés, de

${ }^{49}$ DE GOURCY, Constance :«Introduction : Famille en mouvement : perspectives de recherche », K.Y. (dir.) Familles en mouvement : migration et parentalité en Méditerranée, Publications de l'Université de Provence (31 octobre 2013), Penser le genre, 8 p.

${ }^{50}$ BAROU, Jacques : «Dynamiques de transformation familiale en Afrique subsaharienne et au sein des diasporas présentes en France », Droit et cultures, 73 (2017), 29-47. 
familles issues de la même région ou encore du même pays et, commence à s'inscrire dans un réseau de relations sociales, amicales et pratiques. $»^{51}$. Ainsi, lors d'un entretien, Madame M. décrit ces rapprochements :

«Là-bas dans le 43, il y a les Rifains, des amis à mon mari. Ce sont des gens très bien, quand mon fils qui est en Italie est venu voir son père dans le 43, ils l'ont très bien accueilli. Ils lui ont trouvé un logement au chaud, ils l'ont nourri, ils l'ont accompagné. [...] Tout ça parce que mon mari est une bonne personne. Lui aussi, il a pris soin d'eux quand ils venaient juste d'arriver et qu'ils n'avaient rien. Maintenant, ils ont tous des maisons, des enfants... Deux d'entre eux sont venus nous voir ici, à Montpellier ».

Quoiqu'il en soit, la migration étudiée ici montre que l'enracinement familial (nucléaire) se fait sur le territoire d'origine : le mariage se fait là, les enfants sont conçus et naissent là, la maison familiale se trouve là. Quant à l'enracinement du travailleur, pas du père mais du travailleur, il se fait sur le territoire de résidence, la France, là où se trouvent le travail, les ressources économiques, le logement du travailleur immigré. Parlant de sa famille, Monsieur O. dit :

« Ma famille est toujours à Meknès. Avant de venir en France, je vivais à la campagne où j'étais paysan. Après être venu en France, je me suis marié, on a habité en ville, à Meknès où mes enfants sont nés. Je me suis fatigué en essayant de les ramener mais ça n'a pas marché ! Maintenant, ils ont grandi. Tu vois, la situation n'est pas terrible !»

Evoquant son mari, Madame M. se souvient précisément : « il passait avec nous 25 jours par an et il revenait ici, en France pour travailler. ».

Le congé annuel, mobilité qui chez le travailleur célibataire géographique a pour principale fonction de rendre visite à la famille dans le pays d'origine, permet une jonction temporaire entre ces deux dimensions de la vie de l'immigré et de sa famille. Les allers-retours au pays constituent le seul regroupement physique de tous les membres de la famille, dans le même espace géographique. Ainsi, la fondation même du foyer familial - conception des enfants - se fait autour de la présence temporaire du père et donc essentiellement autour de son absence.

Au cours de notre enquête, nous avons eu un entretien avec la famille $S$. dont le père, décédé en 2011, est venu en France dans les années 1970. Il a été rejoint, à la fin des années 2000,

${ }^{51}$ BOUMAZA, Nadir : (1996), op. cit. 
par sa conjointe et deux de ses filles, avec lesquelles nous avons mené l'entretien. Voici un extrait de nos échanges qui illustre nos propos :

Fille K : Ils se sont mariés, ils ont eu mon frère Hamid [...] Ensuite, il y a eu moi. Moi et mon frère, on est nés au Chleuh. Ensuite, on est partis à Meknès et, peu de temps après mon père a eu un contrat [de travail] de France, je ne sais pas quel âge il avait exactement, à ce moment il devait avoir 26 ans, il était jeune. Il est parti, je venais de naitre, en 1970. Il a organisé ma cérémonie du 7ème jour, ou peut-être même qu'il est parti avant ce jour.

Mère : Non il t'a fait ton baptême et le lendemain matin il est venu en France.

Fille K : Voilà ! Donc il est parti en France et ma mère, moi et mon frère, on est retournés chez le père de ma mère. Comme mon père est parti en France, elle (la mère] est retournée vivre avec ses parents. Quand il est revenu pour la première fois en vacances, on est partis habiter à Meknès. Mon père y a trouvé un logement pour ma mère, la sœur de mon père est restée avec nous et lui, est retourné en France. Ensuite, il y a eu mon frère Khalid qui est né après moi, ensuite H. et ensuite Hakima. On commençait à grandir, l'Hajja [la mère] nous a éduqués, elle a fait le travail de l'homme et de la femme [...]. C'est elle qui nous emmenait à l'école et qui nous en ramenait, elle gérait les problèmes des enfants quand ils commençaient à grandir.

Ainsi, la migration du père fait subir des mutations à cette famille qui est passée du modèle traditionnel élargi, au modèle conjugal puis au modèle célibatairisé. La notion de la famille et de l'espace familial qui en découle, s'inscrivent dans ces mutations.

\subsection{Les rôles sociaux : facteurs d'organisation/réorganisation de la famille}

Comme nous l'avons déjà souligné, les besoins en main-d'œuvre et le marché du travail se caractérisent par leur aspect genré. La quasi-totalité des secteurs d'activités et de recrutement d'immigrés nord et ouest-africains visent des hommes. La politique migratoire fait donc, pendant longtemps, abstraction de leurs familles.

Nous avons également signalé comment ces besoins rencontrent l'aspiration d'hommes jeunes, que les conditions socioéconomiques et politiques de leurs pays d'origine conduisent à se constituer en force de travail en migration. Nous avons rappelé que les 
représentations socioculturelles ${ }^{52}$, elles aussi genrées, assignent aux hommes le rôle de subvenir aux besoins de la famille.

De fait, la construction de l'espace familial va se faire dans la séparation non seulement géographique entre «l'ici » et le «là-bas » mais aussi, en termes de distribution des rôles et des responsabilités au sein de la famille $\mathrm{e}^{53}$. En effet, les migrations et leurs formes « doivent quelque chose aux pratiques et aux identités genrées des migrants et, en retour, la participation au processus migratoire affecte la répartition genrée des tâches et des espaces, les rapports de pouvoir et les identités de genre, et ce d'abord dans le cadre d'observation privilégié qu'est la famille. ${ }^{54}$.Ici, le père est un travailleur immigré célibataire géographique en France. La mère est, elle aussi, une célibataire géographique dans le pays d'origine, responsable d'une famille qui se monoparentalise.

S'agissant des hommes, leur rôle de père est réduit à celui de pourvoyeur d'argent pour la famille. On voit ici comment l'immigration et le système économique dominant limitent leur rôle et leur devoir familial. Par ailleurs, l'accoutumance à la solitude, le fait de ne venir au maximum qu'une seule fois par an, le manque d'habitude de la vie en famille, à diverses sollicitations de la part des proches ou encore à l'agitation des enfants, sont autant de facteurs qui peuvent constituer un engrenage. Celui-ci peut accentuer la perte du rôle de père - autre que celui de pourvoyeur d'argent - et, creuser la distance à la fois temporelle, spatiale, psychologique et affective entre lui et le reste de sa famille.

Les filles de la famille S. expliquent ces difficultés :

Fille $\mathbf{H}$ : L'être humain quand il s'habitue à vivre seul, il ne supporte plus le bruit, les problèmes des enfants... Lui, il venait, il restait avec nous un mois. Il ne restait même pas un mois! C'est rien!

Fille K : La personne vient pour les vacances et repart. Ce n'est pas comme s'il [le père] vivait avec nous. Il ne sait ni qui a mangé ni qui n'a pas mangé.

Fille $\mathbf{H}$ : Il y a des gens, c'est comme ça, quand ils rentrent chez leur famille, ils en ont vite assez : apporte quelque chose à celui-là, donne à celui-ci !... Quand ils retournent en France, ils sont plus tranquilles. Il y a des gens qui sur 10 ans, ne sont

\footnotetext{
${ }^{52}$ Le modèle traditionnel des « Trente glorieuses » est aussi un modèle où l'homme est un bon travailleur et un père de famille d'un point de vue économique.

53 A propos des liens entre migration et rapport sociaux de sexe, voir : MOUJOUD, Nasima :« Effets de la migration sur les femmes et sur les rapports sociaux de sexe : au-delà des visions binaires ", Cabiers $d u$ CEDREF, 16 (2008), 57-79.

${ }^{54}$ RYGIEL, Philippe : «Le genre de l'émigrant(e) et ses transformations », R.P., L.N (dir.), Rapports sociaux de sexe et migration, Publibook, 2007,17-28.
} 
pas retournés une seule fois voir leur famille. 10 ans ! Tu sais ce que c'est ? Pendant 10 ans tu ne retournes pas voir tes enfants!

Fille K : En plus, les enfants grandissent loin de leur père. Il devient comme une personne étrangère. Il n'y a plus cette affection et cette tendresse.

Fille $\mathbf{H}$ : Nous aussi on a ressenti ça.

Fille K : C'était juste notre père, on disait notre père mais on n'a pas vécu avec lui on n'a pas grandi avec lui.

Fille $\mathbf{H}$ : On n'avait pas peur de lui mais il y avait envers lui une grande retenue.

Fille $\mathbf{K}$ : Il y avait une grande retenue, par exemple, on n'osait pas lui demander de l'argent, l'argent on le demandait à notre mère. Il y avait une grande retenue parce qu'on n'avait pas l'habitude avec lui, on n'a pas grandi avec lui, toujours de la retenue avec lui. Il n'y avait pas cette affection paternelle, il était toujours loin de nous, toujours loin de nous!

L'arrivée à la retraite marque aussi un changement dans le statut et le rôle du migrant et dans ses rapports avec sa famille. Elle correspond généralement à une baisse des revenus et, peut aussi être une étape où le migrant connaît une dégradation de son état de santé et une perte d'autonomie. Ces situations peuvent entraîner une dévalorisation symbolique du migrant et du père.

Pour le migrant, la présence en France ne se justifie plus de la même manière puisqu'il ne travaille plus. Certains célibataires géographiques vieillissants justifient maintenant cette présence parla prise en charge médicale. A ce sujet, Monsieur D. témoigne :

\footnotetext{
«Ce n'est même pas une question de médecin, c'est l'hôpital, j'ai un défibrillateur, avec une pile, ici je suis suivi, un suivi médical en cardiologie et en pneumologie, les deux [...] Ah, oui ! Je suis obligé de revenir à chaque fois. Chaque fois que je ne me sens pas bien, je reviens. Je ne pars pas d'ici avant d'avoir pris mes rendez-vous. Et tous les vieux que tu vois ici ne reviennent que pour leurs problèmes de santé, sinon ils n'ont rien à faire ici ».
}

Cette présence peut également être liée aux revenus du retraité lorsqu'ils sont soumis à la condition de résidence. Le témoignage suivant fait ressortir la souffrance que génère cette situation : 
«Ils veulent qu'on reste ici jusqu'à ce qu'on meure. [...] Comment peux-tu rester là 6 mois sans travailler et en ne faisant rien ? 'Tu restes là tu ne vois pas tes enfants. Et toi t'es là, tu restes là. Pourquoi ? Pour que tu gaspilles leur argent ici même. Mais pour que tu le gaspilles avec tes enfants, non! Tu vois ? [...] Si je travaillais, d'accord! Je partirais, je laisserais le travail 1 mois ou 2 et j'irais voir mes enfants puis je reviendrais travailler. Nous, on travaillait entre un congé et un autre. Maintenant qu'on a vieilli, on n'a pas le droit d'aller se poser auprès de nos enfants ? On reste ici jusqu'à qu'ils viennent nous prendre pour la morgue. Ce n'est pas possible ! Ça aussi il faut que vous, les lettrés, vous en parliez. Regarde, je suis en France depuis que je suis jeune, depuis 1972. Là on est en 2014 et ils veulent encore que je reste là. Que tu manges, ou que tu ne manges pas, que tu habites, ou que tu n'habites pas, que tu dors ou que tu ne dors pas, tes enfants là-bas et toi ici ! Ce n'est pas possible ça!»

On voit ici comment la condition de résidence est vécue comme une assignation à résidence, une entrave à la libre circulation et au droit de vivre en famille, par une personne qui se sent légitime, au regard de sa trajectoire professionnelle, pour percevoir une aide sociale non contributive (ASPA).

Outre les questions relatives à la condition de résidence, l'administration fixe le passage à la retraite. Elle peut générer des ruptures de droits et de ressources, de longs délais de traitement, des demandes rejetées et de longues procédures de recours. Toutes ces difficultés rendent difficiles, voire impossibles les déplacements vers la famille et ce, par manque d'argent ou tout simplement par nécessité de surveiller son courrier administratif et d'y être réactif.

Monsieur C. qui, après33 ans de travail dont 22 entant qu'éboueur, se retrouve au chômage quelques années avant sa retraite, suite à un accident du travail qui l'a marqué physiquement. Il enchaine alors les procédures administratives - reconnaissance de l'accident du travail, indemnités journalières liées à l'arrêt maladie, reconnaissance de la maladie professionnelle, chômage, demande de retraite pour inaptitude, revenu de solidarité active en attendant la retraite -pour faire reconnaitre des droits. Il a entamé depuis peu, deux procédures de recours, l'une contre la Caisse Primaire d'Assurance Maladie qui ne lui reconnait pas le caractère professionnel de sa maladie et l'autre, contre la Caisse Nationale d'Assurance Vieillesse qui ne lui accorde pas sa retraite au titre de l'inaptitude au travail. En attendant l'aboutissement de ces procédures et la liquidation de sa retraite, il vit avec le RSA et n'est pas rentré auprès de sa famille depuis 5 ans. 
«Tu travailles toute ta vie et au final tu te retrouves à la rue. J'ai juste de quoi payer mon loyer et rembourser mes dettes. Rien à envoyer à la famille ! [...] Je ne suis pas allé au pays depuis 5 ans maintenant [...] Un ami m’a proposé de me payer le billet d'avion aller/retour mais j’ai refusé. Si je vais là-bas comment je vais faire sur place sans argent? »

Il arrive que le manque d'argent entraine non seulement l'incapacité de subvenir aux besoins de la famille mais aussi l'incapacité matérielle et même psychologique de se déplacer au pays. Ne pas avoir d'argent, c'est être en perte du rôle socioculturel valorisant de chef de famille dévolu à l'homme. La dégradation de l'état de santé et la perte d'autonomie, à cette étape de sa vie, viennent accentuer ce changement de rôle dans la famille.

Un des changements majeurs engendrés par la migration d'hommes seuls, pères dont la famille reste au pays, touche les rôles sociaux de sexe dans la structure familiale. S'agissant des femmes au foyer interrogées, leurs propos font ressortir le lien entre l'éloignement géographique et ce changement de rôles : «Et lui [le mari], il n'était pas là. Je me suis beaucoup sacrifiée pour eux [les enfants], j'ai fait l'homme et la femme. Dieu merci !», dit Madame H.

Notons que cette expression « j'ai fait l'homme et la femme» est commune à de nombreuses mères au foyer, célibatairisées géographiquement par la migration des maris. Ceci témoigne du vécu de ces femmes, cheffes de famille célibataires géographiques, contraintes non seulement de s'occuper, seules, d'enfants mais aussi de jouer des rôles qui traditionnellement sont répartis entre père et mère, tant au niveau de l'éducation des enfants que de la gestion des espaces publics et privés.

Le rôle de ces femmes connait donc une évolution à plusieurs facettes, notamment lorsqu'elles rejoignent leurs maris vieillissants, en perte d'autonomie et/ou en fin de vie dans la migration. Cette migration tardive des épouses permet une nouvelle vie de couple et une relation affective, en France, dans le même logement, sans les enfants, ce qui était jusque-là compliqué par la migration du travailleur immigré et ses contraintes. Néanmoins, quand les conditions de santé du mari sont dégradées et que celles de la conjointe sont meilleures, cette migration peut instaurer un rapport de dépendance du mari vis-à-vis de sa femme qui prend alors en charge notamment les démarches administratives, la gestion du quotidien, les soins.

Ainsi, les statuts et les rôles dictés par des facteurs sociaux, spatiaux et administratifs sont contraignants à plusieurs niveaux et placent la famille dans des configurations complexes, dans un transnationalisme qui l'oblige à se réorganiser en permanence. 


\subsection{Quels rapports en situation d'éloignement et de contrainte?}

Nous avons précédemment souligné comment les dimensions de la vie de l'immigré célibataire géographique et de la vie de sa famille ne sont pas pleinement vécues mais ressenties dans «la double absence $»^{55}$. Cette «double absence » est le résultat d'une situation d'éphémère qui s'inscrit dans la durée.

Cet éphémère éprouvé résulte lui-même des modalités du processus de recrutement, du logement et de la place assignée au travailleur immigré originaire des anciennes colonies $^{56}$. Il découle également de la situation dans les pays d'origine, n'offrant toujours pas de perspectives d'avenir et, plaçant l'immigré et sa famille dans une dépendance à la migration qui au départ devait être provisoire. C'est dans cet éphémère éprouvé que la famille doit organiser ses rapports.

Ainsi, l'éclatement territorial, la discontinuité et les contraintes au niveau, notamment, des pratiques de mobilité, font que les liens avec la famille peuvent être qualifiés de compensatoires et, de redevabilité à la famille et à la collectivité. Ces liens se traduisent à travers l'envoi d'argent ${ }^{57}$ et de cadeaux, les contacts téléphoniques, les envois de photos et de cassettes mais aussi à travers la participation à des projets de développement - construction de routes, d'écoles, de dispensaires, etc. ${ }^{58}$. Ces liens sont également visibles quand l'immigré joue, à distance, le rôle de médiateur dans les conflits familiaux et d'arbitre dans la prise de décisions concernant la famille et la collectivité.

Cependant, le père vit dans un espace que les enfants ne connaissent pas et auquel ils n'ont jamais eu accès. L'histoire des pères et, particulièrement leurs parcours dans la migration restent méconnus par leurs familles. Ainsi, dans beaucoup d'entretiens, nous avons observé la difficulté chez nos interlocuteurs de reconstituer l'histoire familiale. La migration du père vient perturber la mémoire familiale tout en en constituant une partie intégrante. Les rapports avec le père sont souvent basés sur le non-vécu ajouté au non-dit et principalement sur des représentations, ce qui rend problématique la mémoire familiale.

Sur les plans relationnel et affectif, l'éloignement géographique, la courte durée des séjours du migrant travailleur chez sa famille, l'habitude chez lui de vivre seul, isolé ou entre travailleurs sans enfant, compliquent les relations familiales. Nous avons vu comment

\footnotetext{
55SAYAD, Abdelmalek : 1999, 448 p.

56ASSOCIATION DES MINEURS MAROCAINS DU NORD-PAS-DE-CALAIS (AMMN) : 2008. op. cit. ${ }^{57}$ BOLTZ-LAEMMEL, Marie, VILLAR, Paola : « Les liens des migrants internes et internationaux à leur ménage d'origine : portraits croisés de familles étendues sénégalaises », Autrepart, 4, 67-68 (2013), 103-119. ${ }^{58} \mathrm{BA}$, Abdoul Hameth : « Reconstitution territoriale et codéveloppement. L'exemple de la diaspora sénégalaise », Hommes et Migrations, 4, 1286-1287 (2010), 200-211.
} 
des personnes interrogées, les enfants en particulier, décrivent des situations qui expriment l’inadaptation du père à la vie de famille.

Dans un article intitulé «Portrait d'un travailleur migrant: le foyer, une histoire de famille ", Caroline Trouillet ${ }^{59}$, souligne comment la routine d'un père, sur le territoire de résidence, dans le foyer où il habite et qui symbolise « un repère stable », un « sasse intime avec le pays », le place dans l'incapacité d'inscrire sa vie actuelle dans le foyer familial. Ce cas illustre jusqu'à quel point peut aller l'enracinement dans la situation de célibataire géographique. Sans forcément aller jusque-là, cette inadaptation révèle néanmoins un vécu de l'espace familial entre le proche et le lointain. La famille s'habitue de fait à des relations à distance.

L'étape du vieillissement du migrant, particulièrement la maladie et la fin de vie, constitue une épreuve psychologique pour les autres membres de la famille et en particulier pour les enfants qui sont immobilisés dans le pays d'origine et qui ne peuvent pas venir voir leur père légalement en France. Notons certaines répercussions sur les stratégies de mobilité de ses enfants. Pour certains d'entre eux, cette situation du père est un élément déclencheur de l'idée de migrer, même dans la clandestinité, pour le rejoindre et l'accompagner dans ses derniers moments de vie et ainsi reconstruire les liens avec lui.

A la question «quand avez-vous pensé à venir en France ? », la famille S. dont la mère est venue en France légalement et les filles clandestinement, explique :

Fille K : Quand mon père a été très malade et qu'il n'avait personne. Et quand ma mère est venue. C'est-à-dire que si ma mère n'était pas venue, on ne serait pas venues ma sœur et moi. [...] Même si on se disait, je veux partir, lui [le père] il ne voulait pas, donc...

Mère : Lui, ce n'est que quand il est tombé malade qu'ensuite il a demandé le regroupement familial, que l'état m'a donné le visa et ensuite je suis venue. Je suis arrivée et je l'ai trouvé...

Fille K : Oui, voilà ! Il était très malade.

Mère : Que dieu nous préserve, voilà ! il était dans un sale état!

Fille K : Les deux premières années de ma mère en France, comme elle ne savait ni comment aller ni comment venir à chaque fois, elle pleurait.

Mère : Quand je suis arrivée, il avait des béquilles et il ne me montrait pas comment m'orienter dans la ville. Moi je lui disais que cette situation ne me plaisait pas. «Moi je veux faire venir mes filles et ce que tu me dis ne me plait pas ». Et le temps est

59 TROUILLET, Caroline: « Portrait d'un travailleur migrant 2 : Le foyer, une histoire de famille », Africultures, 25 novembre 2012. 
passé et j'ai fait venir K. Un an ou deux après, j'ai fait venir l'autre. Je ne voulais pas la laisser souffrir là-bas.

Fille K : Comme elle n'est pas mariée... Les filles, quand elles ne sont pas mariées, il n'y a personne qui... en plus avec les femmes de mes frères... chacun d'entre eux a sa femme et ses enfants. On vivait dans la même maison. Chacun avec sa femme et ses enfants, qui va se préoccuper de toi ?

La fin de cet extrait laisse entrevoir comment la diminution des revenus de l'immigré retraité et la perspective de leur interruption à sa fin de vie, peuvent engendrer un bouleversement socioéconomique pour certains membres de la famille et constituer un des facteurs de leur migration.

Ces problèmes amènent à constater que de la trajectoire individuelle du père à la trajectoire individuelle de la mère et des enfants, peut s'opérer une reproduction de la précarité dans la migration avec tous ses aspects : statuts administratifs, travail, logement et famille notamment.

\section{Conclusion}

Retenons pour conclure ce qui caractérise la construction de l'espace familial pour les personnes auxquelles nous avons consacré cette réflexion. Partant de notre intérêt pour les migrants âgé-e-s et isolé-e-s nord et ouest-africain-e-s, vivant en France, nous avons été amené à analyser la trajectoire et les pratiques de mobilité de cette population pour en étudier la construction de l'espace familial.

Ainsi, nous nous sommes attaché à contextualiser cette migration pour en dégager les déterminants qui en ont fait une migration d'hommes célibataires géographiques, tout au long de leur trajectoire, depuis leur départ du pays d'origine jusqu'à leur vieillissement dans l'immigration, en passant par leur condition de travailleurs immigrés. A travers cette contextualisation, ce travail a tenté de saisir des aspects historiques et actuel qui ont construit l'isolement de ces générations et qui posent la problématique des mobilités cadrées, de la famille dans le contexte de cette migration d'hommes seuls et de la construction de l'espace familial, en situation de contrainte.

Ainsi, nous avons été conduit à considérer que ce sont les contraintes qui déterminent les pratiques de mobilités et que c'est à partir de ces contraintes qu'on peut 
analyser les capacités individuelles et familiales à mettre en place des stratégies de mobilité et à construire un espace familial.

Partant, nous avons montré comment la famille est travaillée par les questions spatiales, en termes de distance objective et subjective et en termes de pratiques et de rapports à l'espace, marqués par l'éclatement, du fait de cette distance. Ainsi, la migration transforme la famille et l'inscrit dans un processus de perpétuelle organisation/réorganisation spatiale, aux impacts multiples. Nous avons mis en exergue celui qui touche aux rôles et aux rapports sociaux de sexe, en soulignant certains de ses enjeux, non seulement familiaux mais aussi sociaux. Nous avons également fait ressortir la reproduction intergénérationnelle de la précarité dans la migration. Ceci interroge la question de la reproduction des inégalités dans la société. 